\title{
Politisierte Aufsichtsräte? Über die Entwicklung personeller Verflechtungen von Wirtschaft und Politik in Österreich (1989-2011)
}

\author{
Philipp Korom
}

\begin{abstract}
Zusammenfassung: Manager-Parteien-Netzwerke sind Erscheinungen im Zwischenbereich von Wirtschaft und Politik, die die Eigenlogik dieser Systeme in Frage stellen können. Für das Österreich der achtziger Jahre galt, dass der Weg in die Vorstands- und Aufsichtsratsetagen der führenden staatsnahen Wirtschaftsunternehmen nicht an den Großparteien vorbeiführte. Dieser Aufsatz quantifiziert die Entwicklung personeller Verflechtungen von Wirtschaft und Politik in Österreich (1989-2011) und bringt sie mit gesellschaftlichen Machtverschiebungen zwischen der Wirtschaft und dem Staat in Verbindung. Im Fokus der Betrachtung stehen dabei Mehrfachmandatsträger in den Aufsichtsgremien von Österreichs Top-Unternehmen.

Die Analyse zeigt, dass Organisationseliten jene staatsnahen statesmen of industry weitgehend abgelöst haben, die zwischen antagonistischen Interessen der Wirtschaft und des Staates vermittelten. Der Proporz bei Stellenvergaben bleibt jedoch derart stark ausgeprägt, dass ein Regierungswechsel einen „Farbwechsel“ in den Aufsichtsräten führender Unternehmen zur Folge hat. Das politische Kapital hat folglich seine Wertigkeit für das Topmanagement nicht gänzlich verloren.
\end{abstract}

Schüsselwörter: Wirtschaftselite $\cdot$ Politisches Kapital $\cdot$ Österreich $\cdot$ Soziale Netzwerke Parteien

\section{Politicized boards of directors? On the evolution of personal connections between business and politics in Austria (1989-2011)}

\begin{abstract}
Party affiliations of managers form the basis of social networks that are not only positioned between the mostly autonomous systems of politics and business, but are able to undermine the functional logics of these social systems. In the eighties Austrian business was dominated by the state to such an extent that political parties could vote their men of confidence into the boards of large stated-owned companies. This article quantifies the evolution of personal connections between business and politics in Austria (1983-2011) and explains changing patterns by pointing out shifts of power between the state and business. The focus of attention here is on multiple directors in supervisory boards of Austria's big business.
\end{abstract}

(C) Springer Fachmedien Wiesbaden 2014

P. Korom $(\bowtie)$

Max-Planck-Institut für Gesellschaftsforschung,

Paulstrasse 3, 50676 Köln, Deutschland

E-Mail: korom@mpifg.de 
The study shows that businessmen have largely displaced the statesmen of industry who used to mediate conflicts between the state and business within a corporatist setting. "Proporz", however, remains widespread and a change of government still leads to new constellations of political representation in supervisory boards of large companies. Political capital has thus not lost completely its value for top managers.

Keywords: Business elite $\cdot$ Political capital $\cdot$ Austria $\cdot$ Social networks $\cdot$ Parties $\cdot$ Patronage

\section{Einleitung}

Politische Beziehungen öffnen Türen für Unternehmensinteressen - man könnte annehmen, dass dieser Satz zum Standardrepertoire der Soziologie gehört. Tatsächlich haben sich die Sozialwissenschaften bislang kaum mit den hier interessierenden Manager-Parteien-Verflechtungen beschäftigt (für Ausnahmen s. McMenamin und Schoenmann 2007; Stark und Vedres 2012). Diese der Ausdifferenzierung von Politik und Wirtschaft trotzenden Allianzbildungen können prinzipiell durch sehr unterschiedliche Kräfte gesteuert sein.

Im Falle eines Primats der Politik versuchen Parteien, Vertrauenspersonen in Unternehmen einzusetzen, um Wirtschaftsstrategien steuern zu können. Ein Paradebeispiel ist der ehemals große öffentliche Wirtschaftssektor Österreichs, auf den in den siebziger Jahren $25 \%$ des Bruttoinlandsproduktes entfielen (Aiginger 1999). Die damalige Verstaatlichte wurde bis in die achtziger Jahre nahezu ausschließlich durch parteinahe Manager gelenkt. Prominente Beispiele sind der ehemalige Bundesminister für Finanzen (19701981) Hannes Androsch oder der spätere Bundesminister für öffentliche Wirtschaft und Verkehr (1986-1991) Rudolf Streicher.

Werden Märkte wie im Falle Chinas gar staatlich kontrolliert, so ist zu beobachten, dass Manager Beziehungen zur Politik pflegen, um im Wettbewerb Aufträge (z. B. in der Baubranche) oder den Zugang zu knappen Ressourcen (z. B. Kapitalmarkt) erhalten zu können (vgl. Nee und Opp 2007). Im Falle eines ausgewogenen Kräfteverhältnisses oder eines Primats der Wirtschaft nehmen Unternehmen nicht nur über offizielle Verbandstrukturen oder Lobby-Agenturen Einfluss auf die Politik, sondern auch über Spitzenkontakte (Grant 1987). Oftmals hängt ehemaligen Politikern mit Aufsichtsratsmandaten in Bau- und Infrastrukturunternehmen der Ruf nach, sie nützten im Interesse der Unternehmen ihre politischen Beziehungen für Lobby-Aktivitäten. Die frühere Außenministerin Österreichs (2004-2004) Benita Ferrero-Waldner sollen so vor allem ihre internationalen Kontakte für den Aufsichtsratsvorsitz im Baukonzern Alpine qualifiziert haben. Letztendlich kann die Parteinähe von Managern auch von persönlichen politischen Einstellungen herrühren und unabhängig von Organisationsinteressen sein (vgl. Bond et al. 2010; Burris und Salt 1990, S. 350 ff.).

Ein wesentliches Kennzeichen österreichischer Spitzenmanager war ihr politisches Kapital. ${ }^{1}$ Rolf Ziegler untersuchte die Parteienaffiliation einflussreicher Manager, die über mehrere Vorstand- oder Aufsichtsratsmandate in Österreichs größten Unternehmen im Jahre 1976 verfügten. Sein Ergebnis ist eindeutig: „Nur sechs dieser 57 Wirtschafts-

1 In Bezug auf sozialdemokratische Eliten in Skandinavien definierte Bourdieu ,,politisches Kapital“ als eine Spielart des „,sozialen Kapitals“ (Bourdieu 1998, S. 30). 
führer besaßen die politische ,Blutgruppe Null“ oder konnten trotz Recherchen keinem der politischen Lager zugeordnet werden. 17 ließen sich der SPÖ, zwei der FPÖ und 32 der ÖVP zuordnen“ (Ziegler 1987, S. 87).

Der vorliegende Aufsatz geht der Frage nach, ob personelle Verflechtungen zwischen Managern und Parteien nach einer Phase weitreichender Privatisierung und Internationalisierung der österreichischen Volkswirtschaft erodiert sind. Hinterfragt wird außerdem, ob Manager-Parteien-Verflechtungen nach Rückzug des Staates aus der Wirtschaft noch kollektiven Zielen wie der Durchsetzung politischer Wirtschaftsprogramme sowie der Vermittlung zwischen konfligierenden Interessen von Wirtschaft und Staat dienen oder ob sie vielmehr durch Einzelinteressen der Unternehmen oder Parteien motiviert werden. ${ }^{2}$

Da der Beobachtungszeitraum dieser Studie bis 1989 zurückreicht, schildert Abschn. 2 typische Interaktionen zwischen Parteien und staatsnahen Unternehmen zu Zeiten der Verstaatlichten. Es wird auf Privatisierungen im Banken- und Industriewesen eingegangen, die u. a. zu einem Wandel der Managerprofile führten. Abschn. 3 diskutiert die Funktionen von Manager-Parteien-Verflechtungen. Im nachfolgenden empirischen Abschnitt werden die Parteienaffiliationen der wichtigsten Aufsichtsräte österreichischer Großunternehmen im Längsschnitt betrachtet. Der Schlussteil diskutiert, warum trotz des zunehmenden Elitenpluralismus das politische Kapital ein Kennzeichen von Teilen des Spitzenmanagements geblieben ist.

\section{Vom Parteienproporz zum Elitenpluralismus - Manager-Parteien- Verflechtungen in Österreich (1945-2011)}

\subsection{Parteienproporz und Elitenkartelle - Die Personalpolitik in Österreichs Wirtschaft der Nachkriegszeit (1945-1986)}

In Österreichs Wirtschaft spielte der Staat im 20. Jahrhundert stets eine gewichtige Unternehmerrolle. Schon im Zuge der Weltwirtschaftskrise von 1929 kam es zur Verstaatlichung der mit dem Großteil der österreichischen Industrie aufs engste verbundenen Bank Creditanstalt (CA). Mit den Verstaatlichungsgesetzen von 1946/1947 wurden 70 Unternehmen - darunter drei Kreditinstitute, die wiederum 61 Großbetriebe als Beteiligungen hielten - und somit ein Fünftel des österreichischen Industriepotentials (Turnheim 2009) zu Eigentum der öffentlichen Hand erklärt. Noch Mitte der achtziger Jahre verfügte Österreich über den proportional größten öffentlichen Wirtschaftssektor Westeuropas. Die „Austrifizierung“ der Wirtschaft geht auf eine rechtliche und staatspolitische Zwangslage zurück: Das Potsdamer Abkommen (1945) sah vor, dass deutsches Eigentum als Wiedergutmachung den Alliierten zufallen sollte. In der Verstaatlichung sah man daher die einzige Möglichkeit, wichtige Produktionsstätten in Österreich zu halten.

Die Verstaatlichte unterstand anfangs der Regierung, zunächst repräsentiert durch das Ministerium für Vermögenssicherung und Wirtschaftsplanung, später durch das Verkehrsministerium und das Bundeskanzleramt. Bis in die achtziger Jahre bestand sie aus

2 Streeck und Thelen (2005) bezeichnen den Prozess, bei dem bestehende Institutionen (oder soziale Beziehungen) für einen den gegenwärtigen Umständen eher angemessenen Zweck genutzt werden als conversion. 
drei großen Wirtschaftsbereichen (vgl. Meth-Cohn und Müller 1994, S. 160): der mit der treuhändigen Ausübung der Anteilsrechte der Republik an den verstaatlichten Unternehmen beauftragten Dachorganisation ÖIAG ${ }^{3}$; den Großbanken CA und Länderbank und deren Industriebeteiligungen sowie dem Energiesektor. Noch um 1980 stand die ÖIAG den größten Industrieunternehmen vor, darunter der Voest-Alpine (Eisen- und Stahlproduktion), der Chemie Linz (Chemieindustrie), der Elin-Union (Elektrotechnik), der OMV (Ölindustrie), den Vereinigten Metallwerken Ranshofen-Berndorf (Aluminiumindustrie) sowie den Simmering-Graz-Pauker-Werken (Maschinenindustrie). Diese und andere Unternehmen wurden in den Dienst der Wachstumspolitik gestellt und sicherten Arbeitsplätze - darin bestand ihr gesamtwirtschaftlicher Auftrag. ${ }^{4}$ In den ersten zwanzig Jahren hat die Verstaatlichte erheblich ,,zu Dynamik und Stabilität der Industrieentwicklung beigetragen und die heimische Wirtschaft ausreichend und billig mit Grundstoffen versorgt [...] bis in die späten 70er Jahre hatte sie ein höheres Umsatzwachstum als der Industriedurchschnitt, wenn auch mit sinkenden Erträgen“ (Aiginger 1991, S. 517). Alle verstaatlichten Unternehmen wurden grundsätzlich privatwirtschaftlich geführt. „Der sichtbare Erfolg einer marktorientierten Wirtschaftsweise reduzierte die Chancen staatlicher Lenkung“ (Mathis 1995, S. 172). In den Staatsbetrieben agierte das Management - sieht man von Krisenzeiten ab - weitgehend unabhängig. Seit dem Kompetenzgesetz 1956 hatten die Geschäftsführer der IBV (vgl. Fn. 2) jedoch das gesetzlich verankerte Recht, Aufsichtsräte und Geschäftsführer zu berufen, wobei nach $\S 7$ des Gesetzes ,,das Kräfteverhältnis und die Vorschläge der im Nationalrat vertretenen politischen Parteien zu berücksichtigen“ waren. „Damit hat der Staat sein wesentlichstes Besitzrecht, das allein ihm die Möglichkeit zu einer Einflussnahme auf Führung und Verwaltung seiner Unternehmungen geben könnte, an die politischen Parteien delegiert" (Koren 1964, S. 108). Der gesetzlich verankerte Proporz führte dazu, dass jede Partei ihre Vorstands- und Aufsichtsratspfründe verwaltete. Aufgrund des gegenseitigen Misstrauens der Parteien war einem ,roten“ Aufsichtsrat stets ein „schwarzer“ zur Seite gestellt. Bei parteinahen Spitzenmanagern konnte es sich durchaus um kompetente Führungskräfte handeln, ihre Nominierung war dennoch stets von ihrer Nähe zu einer der beiden Großparteien abhängig. Die Folge war eine kartellartige Verteilung von Managerpositionen, die von Beginn an heftig kritisiert wurde (vgl. Stiefel 2011, S. 137 f.).

Eine gewisse Aufweichung des Proporzes erfolgte Anfang der siebziger Jahre als (zumindest offiziell) parteiungebundene Personen, sog. „Blutgruppe-Null“-Manager, in Aufsichtsräte berufen wurden (Dobler 1983). Von einem Rückgang des Proporzes kann

3 Die ÖIAG hatte mehrere Vorläuferinnen: 1956 setzte die ÖVP erstmals eine gesellschaftsrechtliche Trennung durch. Für die Verstaatlichte war nicht mehr das Ministerium direkt zuständig, sondern die IBV (Industriebeteiligungsverwaltung) als eine Abteilung des Ministeriums. 1956 wurde die IBV von der ÖIG (Österreichische Industrieverwaltungs-GmbH) abgelöst, die die Eigentumsrechte des Bundes verwalten sollte. Mit dem Wahlsieg der SPÖ 1970 wurde die ÖIG schließlich in die ÖIAG überführt.

4 Der gesamtwirtschaftliche Auftrag wurde u. a. im Jahr 1970 bei der Gründung der ÖIG durch die ÖVP-Alleinregierung erneuert. Die Anteilsrechte des Bundes an den verstaatlichten Unternehmen, so hieß es im $\S 1$ des ÖIG-Gesetzes, seien so auszuüben, ,wie es das Wohl der Gesellschaften unter Berücksichtigung der Interessen der Gesellschafter und der Arbeitnehmer dieser Gesellschaften sowie der gesamten Volkswirtschaft erfordert“ (Weber 2011, S. 133). 
erst ab Mitte der achtziger Jahre gesprochen werden, als offensichtlich wurde, dass dieses System der Stellenbesetzung ein offensichtliches Hindernis für die Lösung der mit der Verstaatlichen Industrie zusammenhängenden Probleme darstellte.

\subsection{Das Ende der parteipolitischen Einflussnahme? - Privatisierung der österreichischen Wirtschaft (1987-2011)}

Dem internationalem Trend folgend setzten in den achtziger Jahren umfangreiche Privatisierungsvorhaben der von mehreren Skandalen erschütterten Verstaatlichten auch in Österreich ein. Führte der aufgedeckte, gegen das Bundesgesetz verstoßende Waffenexport der Voest-Tochter Noricum in den Iran lediglich zu politischen Querelen, so folgte 1985 ein ökonomisches Erdbeben, als publik wurde, dass Intertrading - ein weiteres Tochterunternehmen des Voest-Alpine-Konzerns - bei Baisse-Spekulationen am Ölmarkt Verluste in Höhe von 5,7 Milliarden Schilling (414 Mio. €) erlitten hatte. Schon zuvor war die Verstaatlichte infolge der weltweiten Branchenkrise im Bereich der Eisen- und Stahlindustrie, missglückter Diversifizierungen und permanenter politischer Interventionen im Sinne der Beschäftigungspolitik (und damit sinkender Konkurrenzfähigkeit) zu einem ständigen Subventionsempfänger des Bundes geworden (vgl. Stiefel 2011, S. 167 ff.). Die steigenden Verluste staatlicher Betriebe seit der zweiten Hälfte der Ära Kreisky waren jedoch nur einer der vielen Gründe eines Richtungswechsels in der Wirtschaftspolitik. Als weitere Anstöße führen Belke und Schneider (2006) an: der Anreiz, mit den Erlösen das Staatsbudget aufzubessern; Lobbying von nationalen und mehr noch von internationalen Investmentbanken; eine steigende Akzeptanz der Idee von ,,mehr privat, weniger Staat" in der Bevölkerung sowie eine zunehmend positive Haltung gegenüber Privatisierungen bei allen Parteien. Zwischen 1986 und 2003 verkaufte der Staat schrittweise Anteile an solchen Großunternehmen wie AMAG, Voestalpine, VA Stahl, VA Technologie, Böhler Uddeholm, AT \& S, Austrian Airlines oder Austria Tabak (Turnheim 2009; Obinger 2006). Am Ende stand jeweils die Vollprivatisierung. Gegenwärtig ist die Republik Österreich nur mehr an dem Mineral- und Chemiekonzern OMV (31,5\%), der Österreichischen Post (52,85\%), der Telekom Austria (28,42\%) und der GKB-Bergbau $(100 \%)$ indirekt über die ÖIAG beteiligt. Direkte Eigentümerin ist die Republik im Falle der Österreichischen Bundesbahnen (ÖBB) und des bundesweiten Elektrizitätskonzerns „Verbundgesellschaft“, der wiederum an den Landesenergieversorgern (EVN, KELAG, TIWAG usw.) mehrheitlich beteiligt ist. Eine aktuelle Studie sieht in diesen beiden Wirtschaftsbereichen die größten zukünftigen Privatisierungspotentiale. Allein ein Rückgang der öffentlichen Hand aus allen Energieversorgungsunternehmen auf die aktienrechtliche Sperrminorität $(25 \%+1$ Aktie) würde einen geschätzten Verkaufserlös zwischen 3,2 und 6,3 Mrd $€$ erbringen (Böheim et al. 2010).

Die durch die Privatisierung herbeigeführte weitgehende Entkopplung von Wirtschaft und Politik hat zu einer Abwertung des politischen Kapitals im Spitzenmanagement Österreichs geführt. Beschreibt sich der derzeitige Präsident der Österreichischen Nationalbank (ÖNB), Claus J. Raidl (geb. 1942), noch als „Produkt des Proporzes“ (Der Standard 2011a), so machte der Vorstandsvorsitzende der Erste-Banken, Andreas Treichl (geb. 1952), 2011 Schlagzeilen, als er im Zusammenhang mit staatlich reglementierten Krediten Politiker öffentlich als „blöd und feig“ (Der Standard 2011b) beschimpfte. Was 
hier vor allem zum Ausdruck kommt, ist eine Verschiebung der Machtbalance. Spitzenmanager sind der Willkür von Parteien weniger ausgesetzt, ihr Stellenwert ist aufgewertet - nur aus dieser Position ist eine derartige öffentliche Schelte möglich.

Des Weiteren ist zu berücksichtigen, dass Parteien insgesamt an Rückhalt in der Bevölkerung verloren haben. SPÖ und ÖVP sind im engeren Sinne keine Massenparteien mehr: „1994 betrug der Organisationsgrad (Mitglieder einer Partei als Prozent ihrer WählerInnen) bei der SPÖ noch knapp 32\%, bei der ÖVP knapp 34\%" (Pelinka und Rosenberger 2000, S. 133). Es ist daher naheliegend anzunehmen, dass die Parteienmitgliedschaften im Management abgenommen haben. Der vor allem durch zahlreiche Korruptionsskandale ${ }^{5}$ entstandene Ansehensverlust der politischen Klasse in Österreich führt drittens dazu, dass Talente in viel stärkeren Ausmaß Karrieren in der Wirtschaft als in der Politik anstreben.

Trotz Machtverschiebungen, Mitgliederrückgang der Großparteien und einem Prestigeverlust der Politik ist beim Management von heute oftmals eine Parteinähe zu erkennen. Der bereits erwähnte Spitzenmanager Andreas Treichl war etwa von 1991 bis 1997 Finanzreferent der ÖVP. Der Wechsel des ehemaligen Finanzministers Josef Pröll in den Vorstand des Raiffeisen-Mischkonzerns Leipnik-Lundenburger machte jüngst die guten Beziehungen zwischen ÖVP und der Raiffeisen-Bankengruppe deutlich. Glaubt man der Presse, so feiert der Proporz in den staatsnahen Unternehmen weiterhin fröhliche Urstände.

Diese Beobachtung begründet das Interesse, den Grad der Manager-Parteien-Verflechtung in einer Längsschnittbetrachtung (1989-2011) zu erfassen. Neben der reinen Quantität soll auch die (gewandelte) Bedeutung dieser Querverbindungen untersucht werden.

\section{Bedeutungen und Wandel von Parteien-Manager-Verflechtungen in Österreich}

Sind Parteien-Manager-Verflechtungen nachgewiesen, so präsentieren sie sich der Sozialwissenschaftlerin zunächst einmal als die sprichwörtliche black box. Für jeden Einzelfall muss rekonstruiert werden, wie der Kontakt zur Politik beschaffen ist und welche „Geschichte“ mit dem Netzwerk verbunden ist. Denn ,social networks are phenomenological realities, as well as measurement constructs. Stories describe the ties in networks (...) A social network is a network of meanings" (White 1992, S. 65-67, eig. Hervorh.). Unter Berücksichtigung von „stories“ können drei Typen unterschieden werden:

\subsection{Machtasymmetrische Verflechtungen}

Die Politisierung der Aufsichtsräte kann Ausdruck der für Österreich typischen Proporzdemokratie sein. Parteien tendierten dazu, „sich jedes organisierbare Interesse auch organisatorisch einzuverleiben“, was zur Folge hat, „dass nahezu alle Gliederungen der

5 Einen Überblick über politische Korruption in der Zweiten Republik gibt Sickinger (2011). Fehlende Antikorruptionsgesetze öffneten in Österreich Bestechung, Vorteilsnahme und illegale Parteifinanzierung Tür und Tor. Erst 2012 wurde das Korruptionsstrafrechtsänderungsgesetz vom Nationalrat beschlossen. 
Gesellschaft die Polarität der politischen Organisationen widerspiegeln“ (Kafka 1962, S. 595). Das Prinzip gegenseitiger Machtaufteilung sollte konflikthemmend auf die Beziehungen zwischen dem sozialistisch-laizistisch-städtischen und dem konservativkatholisch-ländlichen Lager in Österreich wirken. Im Bürgerkrieg von 1934 waren die Auseinandersetzungen zwischen diesen Gruppierungen eskaliert. Die Zweite Republik beschloss man unter der jeweiligen Kontrolle des anderen politischen Lagers aufzubauen. Der Proporz im ÖIAG-Bereich war rechtlich verankert, Bundeskanzler und Bundesregierung hatten bei der Entsendung der Aufsichtsratsmitglieder der ÖIAG die proportionale Stärke der politischen Parteien im Parlament zu berücksichtigen. Für die Wahl und Abberufung der Aufsichtsräte in den ÖIAG-Töchtern bedurfte es wiederum der Zustimmung von 2/3 der abgegebenen Stimmen des ÖIAG-Aufsichtsrats (Fehr und Van der Bellen 1982, S. 126). Obwohl es keine rechtlichen Bestimmungen für Personalbestellungen bei den beiden verstaatlichten Großbanken (Creditanstalt, Länderbank) gab, stellte sich auch dort eine Proporztradition ein.

Mit parteipolitischer Ämterpatronage ist heute noch in allen Unternehmen zu rechnen, die sich mehrheitlich im Besitz des Bundes oder einzelner Länder befinden. Nicht anders als in der Privatwirtschaft bestimmen dort die Eigentümer über Aufsichtsratsbestellungen.

Der Einfluss von Parteien auf Unternehmensentscheidungen durch derartige Ämtervergaben dürfte verschwindend gering sein. Das hat zumindest zwei Gründe: Erstens ist die Tauschbeziehung zwischen Patron (Partei) und Klient (Manager) diffus, d. h. gegenseitige Pflichten sind nicht definiert. „Im Unterschied zum Vertragsmodell beruht die Gegenleistung nicht auf technisch-juristischen Mechanismen, sondern auf dem Gefühl der persönlichen Verpflichtung“ (Müller 1988, S. 460). In einem kompetitiven Umfeld, das eine strenge Ausrichtung nach betriebswirtschaftlichen Kriterien erfordert, können sich Manager leichter von Gegenleistungen befreien. Zweitens ist der Proporz selbst als Kontrollmechanismus zu sehen, der seine Wirkung dadurch entfaltet, dass ,parteipolitische Entscheidungen den Widerstand der jeweils anderen Partei hervorrufen“" (Fehr und Van der Bellen 1982, S. 128).

\subsection{Machtsymmetrische Verflechtungen I - Interessenbasierte Netzwerke}

Anders verhält es sich mit Verflechtungen, in denen keiner der Involvierten die Beziehung tendenziell dominieren kann. Dieses Beziehungsgeflecht ist in manchen Fällen durch Interessen geprägt: Bei den Unternehmensinteressen eines Spitzenmanagers kann es sich u. a. um den direkten Informationszugang zu Ausschreibungen der öffentlichen Hand, das Offenhalten rascher Interventionsmöglichkeiten auf geplante Reformen des Wirtschafts-, Steuer-, Zoll-, Kartellrechts usw. handeln. Von Parteiinteresse kann es sein angesichts der geballten ökonomischen Macht einzelner Unternehmen(-sgruppen), möglichst gute Managerbeziehungen zu pflegen, um so Beschäftigung, Investitionen, Außenhandel im Sinne der Partei beeinflussen zu können. Das Eigeninteresse einer Partei an einer engen Beziehung zu einem Manager kann auch davon herrühren, dass gelegentlich Positionen zu besetzen sind, die in der Wirtschaft gesammeltes Erfahrungswissen erfordern (,,personelle Reserve"). Eine derartige machtsymmetrische Manager-Parteien-Verflechtung liegt etwa im Fall des früheren Generaldirektor der Raiffeisenlandesbank OÖ Ludwig Scharinger und der ÖVP vor. Scharinger (,König Ludwig“) wird nachgesagt, dass er innerhalb 
des Bundeslandes Oberösterreich mehr Einfluss (gehabt) habe als der oberösterreichische Landeshauptmann selbst. Seine Machtbasis hat der Manager alter Schule durch eine massive Beteiligungspolitik ausbauen können. Die RLB OÖ hält derzeit direkte oder indirekte Anteile an 528 (zumeist oberösterreichischen) Unternehmen, was zur Konsequenz hat, dass in Oberösterreich die RLB das Wirtschaftsgeschehen dominiert. Als Scharinger, der einer katholischen, farbentragenden Studentenverbindung (Cartellverband) angehört, 2012 in Pension ging, nominierte ihn die ÖVP für einen Aufsichtsratsposten in der ÖBB. Während seiner Managertätigkeit war es Scharinger u. a. aufgrund seiner guten Kontakte zur Politik gelungen, die Schüssel-Regierung davon zu überzeugen, dass die Republik das Gros der Aktien an Österreichs größtem Stahlkonzern, der Voestalpine AG, an ein von der RLB OÖ angeführtes Konsortium (und nicht an internationale Investoren) verkaufen solle. Die Zeitschrift „The Economist“ (2003) zog damals folgendes Resümee: „Mr. Schüssel, who talks fondly of open borders and free markets, now sounds happy to accept a much lower price for Voestalpine to keep it in familiar hands“. Interessegeleitet ist diese Verflechtung für beide kollektiven Akteure, die Partei und das Unternehmen. Die Partei hält durch ihre guten Manager-Beziehungen Kontakt zu dem mächtigsten Wirtschaftsunternehmen des Landes, das Unternehmen verspricht sich von seiner sozialen Einbettung Wettbewerbsvorteile. Typisch für derartige Verflechtungen ist nicht nur eine gewisse Institutionalisierung der Beziehung (Parteimitgliedschaft), sondern auch kontinuierliche Beziehungsarbeit in Form von Bekundungen gegenseitiger Anerkennung. ${ }^{6}$

\subsection{Machtsymmetrische Verflechtungen II - Der Manager als politische Person}

Des Weiteren können Manager auch als Privatpersonen und nicht als Organisationsangehörige deklarierte politische Haltungen einnehmen und die Nähe zu Parteien suchen. In diesen Fällen verläuft die Managerkarriere typischerweise gänzlich in der Privatwirtschaft, und es gibt wenig Grund anzunehmen, dass die Parteinähe unternehmerische Vorteile einbringen könnte. Über den Vorstandsvorsitzenden von Spar Österreich AG, Gerhard Drexel, ist etwa bekannt, dass er Mitglied des ÖVP-nahen Cartellverbands ist. Weder die Eigentümerstrukturen noch der Kundenmarkt des Handelsunternehmens Spar legen nahe, dass Drexel als Organisationsangehöriger der ÖVP nahe steht.

Im Zuge der Privatisierungen in der österreichischen Wirtschaft haben die Parteien erheblich an Patronageressourcen eingebüßt. Es ist daher für Parteien zunehmend schwierig, Patron-Klient-Beziehungen als sozialen Tausch zu konstituieren. Zu erwarten ist daher eine Zunahme interessegeleiteter oder ins Private reichender Kontakte zwischen Managern und Parteien (s. Kap. 3.2 und 3.3) bzw. eine conversion (Streeck und Thelen 2005) bestehender Verflechtungen: Jene Manager, die in staatsnahen Unternehmen nur mit einem Parteibuch Karriere machen konnten, sind nach dem Rückzug des Staates aus der Wirtschaft von parteipolitischen Fesseln befreit. Bleibt der Manager erfolgreich

6 Diese können vielerlei Formen annehmen. Um nur ein Beispiel zu nennen: Zwei führende Raiffeisenbanker (Christian Konrad, Erwin Hameseder) sind dem Personen-Komitee „Wir-Pröll““ beigetreten, das den ÖVP-Politiker Erwin Pröll in den niederösterreichischen Landtagswahlen 2008 öffentlich unterstützte. Im Rahmen der Verabschiedung des Obmanns der Raiffeisen Holding NÖ-Wien wurde Erwin Pröll wiederum 2012 aufgrund seiner Verdienste um das Bundesland die Raiffeisen-NÖ-Wien-Nadel erster Klasse verliehen. 
und loyal gegenüber der Partei, so ist zu erwarten, dass ihm Angebote für frei werdende Positionen unterbreitet werden. Diese kann er jedoch aufgrund seiner neu gewonnenen Autonomie jedoch auch ablehnen.

\section{Methode}

\subsection{Die Erhebung der Parteinähe von Managern}

Empirische Erhebungen zur Parteinähe von Managern haben stets mit der Non-Response-Problematik zu kämpfen. Manager geben zu ihren Kontakten in die Politik ungern Auskunft. Sie möchten alleine an ihren wirtschaftlichen Erfolgen gemessen werden. Für die USA misst man die Parteinähe indirekt anhand der Zahlungen der Großunternehmen an Political Action Committees (PACs) (vgl. Mizruchi 1992). Das sind Lobbygruppen, die gewisse Regierungsmitglieder unterstützen oder bekämpfen. In Deutschland können Beziehungen zwischen Unternehmen und Parteien durch offengelegte Parteispenden nachvollzogen werden (vgl. Höpner 2006). Auf derartiges Datenmaterial kann im Falle Österreichs nicht zurückgegriffen werden. Als einzige nonreaktive Erhebungsmethode kommt die Inhaltsanalyse von Zeitschriftenartikeln in Frage (vgl. Ennser-Jedenastik 2013). In der Presseberichterstattung bringen Journalisten Manager aus unterschiedlichen Gründen mit einer Partei in Verbindung. Sie berichten über die Parteizugehörigkeit eines Managers, verweisen auf politische Karrierestationen, unterstellen eine ideologische Nähe zwischen Manager und Parteiprogramm oder schreiben über einen Fall von politischer Postenschacherei.

Für die Recherche bestehender Parteienaffiliationen von Managern hat sich das Pressearchiv der APA (Austria Presse Agentur), das für einen Zeitraum von über 40 Jahren (2012-1970) die gezielte Suche von Managernamen in sämtlichen österreichischen Tageszeitungen, Zeitschriften und Magazinen ermöglicht, als besonders hilfreich erwiesen. Wurde in zumindest drei Presseartikeln von der Politiknähe eines Managers berichtet, so ist dieser Aufsichtsrat entweder der ÖVP oder der SPÖ zugeordnet worden. ${ }^{7}$ Der Erhebung liegt ein Sample von insgesamt 215 Zeitungsartikeln zugrunde.

Den auf diese Weise erhobenen Verbindungen kann keine spezifische Bedeutung zugemessen werden. In der Netzwerkanalyse ist es Standard, relationale Merkmale derart zu erheben, dass die Angabe von A, es liege eine X-Beziehung zu B vor, mit der entsprechenden Angabe von B in Bezug auf A verglichen wird. Erst bei einer entsprechenden Übereinstimmung kann ein Beziehungsnetz konstruiert werden. Für die vorliegende Untersuchung war jedoch eine derartige Vorgehensweise nicht umsetzbar.

7 In den wenigen historischen Fällen, in denen nur ein oder zwei einschlägige Artikel ausfindig gemacht werden konnten, wurden weitere biographische Recherchen angestellt. Falls dabei eindeutige Hinweise auf Parteinähe ausgemacht werden konnten (z. B. die Mitgliedschaft eines Managers in dem mit der Sozialdemokratie sympathisierenden Wiener Wirtschaftsklub), wurde auch in diesen Fällen die Parteinähe entsprechend kodiert. 


\subsection{Die Stichprobe: Multiaufsichtsräte}

Die empirische Erhebung berücksichtigt jene Aufsichtsräte, die dem sogenannten inner circle (Useem 1984) der österreichischen Wirtschaft zu unterschiedlichen Zeitpunkten angehörten, womit an die Vorgängerarbeit von Ziegler (1987) angeschlossen wird. Useem versteht darunter den innersten Kern des Kontaktnetzwerkes der Wirtschaftselite, das durch die Netzwerkvirtuosen unter den Top-Managern der größten Unternehmen aufgespannt wird. Diese Verbindungsmänner üben stets mehrere Aufsichtsratsmandate in unterschiedlichen Unternehmen aus (Multiaufsichtsräte). Unter Bezugnahme auf die USA und England stellte Useem fest, dass diese Personen den engen Interessenhorizont von Unternehmen und Branchen transzendieren und so das Wirtschaftssystem insgesamt koordinieren sowie Brücken zu anderen Gesellschaftsbereichen wie der Politik bauen können. Für Österreich kann mit Sicherheit gesagt werden, dass Multiaufsichtsräte in der Regel höchst erfolgreiche Manager sind und über große, zumeist den Wirtschaftsbereich überschreitende Kontaktnetzwerke verfügen.

Um eine repräsentative Stichprobe aus Österreichs Unternehmenslandschaft zu ziehen, wurde das Verhältnis der 500 größten Unternehmen aus den Branchen Industrie, Handel und Dienstleistungen für mehrere Jahrgänge anhand der Unternehmensrankings des Wirtschaftsmagazins „Trend“ berechnet. Das eruierte Verhältnis von 7 (Industrie): 2 (Handel): 1 (Dienstleistungen) wurde auf die 100 umsatzstärksten Unternehmen des Landes angewandt (70:20:10). Zusätzlich zu diesen Unternehmen wurden aus dem Finanzsektor stets die 20 Banken mit den höchsten Bilanzsummen des Jahres sowie die „Top 5“ der Versicherungsunternehmen nach Prämieneinnahmen berücksichtigt. Für die insgesamt 125 Unternehmen jedes Jahrgangs wurden zwei Managementebenen vollständig erfasst: Vorstand/die Geschäftsführung und Aufsichtsrat. Ein derartiges Sample wurde für sechs Jahre erhoben (vgl. Tab. 1). Eine vergleichende Analyse dieser Augenblicksaufnahmen soll es ermöglichen, langfristige Dynamiken in der Verflechtung von Wirtschaft und Politik sichtbar zu machen.

In Tab. 1 sind insgesamt 199 Multiaufsichtsräte enthalten, die oftmals aufgrund kontinuierlicher Karrieren in den einzelnen Jahrgängen mehrmals berücksichtigt werden. Die Tabelle macht deutlich, dass die Anzahl an Netzwerkvirtuosen im Zeitverlauf kontinuierlich abnimmt, um sich ab 2008 auf einem relativ konstanten Niveau zu stabilisieren. Dieser Befund stimmt weitgehend mit den Ergebnissen einer Analyse der Unternehmensnetzwerke innerhalb der größten 250 Firmen in Österreich (1976-2008) überein, die zeigt, dass die Privatisierung und Internationalisierung der österreichischen Wirtschaft nur zu einer relativen Abnahme der Verflechtungen, jedoch nicht zu einem kompletten Kohäsionsverlust geführt hat (vgl. Korom 2012).

Im inner circle wurden ab 1999 Netzwerkvirtuosen mit mehr als sechs Positionen (Schmidt-Chiari, Randa, Grünwald) ${ }^{8}$ zunehmend die Ausnahme - das spricht für die

8 Guido Schmidt-Chiari sowie Gerhard Randa sind die ehemaligen Generaldirektoren der Creditanstalt bzw. der Bank Austria, die in deren Industriebeteiligungen und innerhalb des Bankensektors zahlreiche Aufsichtsratsmandate akkumulierten. Oskar Grünwald war Vorstandsvorsitzender der ÖIAG und damit in zahlreichen Aufsichtsräten der diversen ÖIAGTöchter (OMV, Siemens Österreich u. a.) vertreten. 
Tab. 1: Manager mit mindestens 3 Mandaten (AR, Vors.) in den größten 125 Unternehmen Österreichs nach Anzahl der Geschäftspositionen (1989-2011)

\begin{tabular}{lcccccc}
\hline & $\begin{array}{l}1989 \\
\mathrm{abs}(\%)\end{array}$ & $\begin{array}{l}1992 \\
\mathrm{abs}(\%)\end{array}$ & $\begin{array}{l}1999 \\
\mathrm{abs}(\%)\end{array}$ & $\begin{array}{l}2004 \\
\mathrm{abs}(\%)\end{array}$ & $\begin{array}{l}2008 \\
\mathrm{abs}(\%)\end{array}$ & $\begin{array}{l}2011 \\
\mathrm{abs}(\%)\end{array}$ \\
\hline $\begin{array}{l}\text { 8 Positionen } \\
\text { o. mehr }\end{array}$ & $3(4,8)$ & $2(3,4)$ & $5(8,9)$ & $0(0,0)$ & $0(0,0)$ & $0(0,0)$ \\
7 Positionen & $0(0,0)$ & $2(3,4)$ & $2(3,6)$ & $1(2,0)$ & $0(0,0)$ & $2(5,7)$ \\
6 Positionen & $3(4,8)$ & $3(5,2)$ & $2(3,6)$ & $4(8,2)$ & $2(5,7)$ & $2(5,7)$ \\
5 Positionen & $7(11,1)$ & $6(10,3)$ & $6(10,7)$ & $4(8,2)$ & $1(2,9)$ & $1(2,9)$ \\
4 Positionen & $20(31,7)$ & $10(17,2)$ & $10(17,9)$ & $15(30,6)$ & $11(31,4)$ & $7(20,0)$ \\
3 Positionen & $30(47,6)$ & $35(60,3)$ & $31(55,4)$ & $25(51,0)$ & $21(60,0)$ & $23(65,7)$ \\
$\sum$ Manager & 63 & 58 & 56 & 49 & 35 & 35 \\
$\sum$ Positionen & 242 & 226 & 233 & 186 & 124 & 128 \\
\hline
\end{tabular}

Professionalisierung des Kontrollorgans. ${ }^{9}$ Ausnahmen gibt es in den nachfolgenden Jahren dennoch. Dabei handelt es sich um die Raiffeisen-Banker Konrad, Hameseder, und Rothensteiner, die u. a. mehrere Aufsichtsratsmandate in Tochterunternehmen der eigenen Geschäftsgruppe hielten. ${ }^{10}$ Die Multiaufsichtsräte von heute bekleiden zumeist nur noch drei oder vier Managementpositionen.

Im Folgenden soll untersucht werden, inwiefern Parteinähe innerhalb dieses ausgewählten Personenkreises eine Rolle spielt.

\section{Ergebnisse}

\subsection{Fortsetzung des Proporz trotz Rückgang von Multiaufsichtsräten}

In dem Untersuchungszeitraum zwischen 1989 und 2011 wurde die Regierung (Vranitzky II-V, Klima I) bis 2000 von den Sozialdemokraten angeführt. Koalitionspartner war jeweils die ÖVP. Zwischen Februar 2000 und 2007 regierte das Kabinett Schüssel I und II (ÖVP) mit der Beteiligung der FPÖ und des BZÖ. Schließlich kam es ab dem Jänner 2007 wieder zu einer großen Koalition unter der Führung der Sozialdemokraten (Gusenbauer I, Faymann I). Die Regierungsumbildung von 2000 wird oftmals als „Wende“ bezeichnet, da sie die langjährige Kompromisspolitik zwischen den Großparteien SPÖ und ÖVP beendete. Im wirtschaftspolitischen Bereich griff verstärkt eine neoliberale Ideologie Platz, die zu verstärkten Privatisierungen unter dem Motto „mehr privat, weniger Staat“ führte. Eine der Besonderheiten der Wirtschaftspolitik der ÖVP-FPÖ-Koalition waren

9 Es sei angemerkt, dass der Aufsichtsrat ab den 1990er Jahren zunehmend mitunternehmerische Verantwortung übernimmt. So zählt seine Stimme z. B. im Falle von ,genehmigungspflichtigen Geschäften“ wie der Errichtung oder Schließung von Zweigniederlassungen. Das Gesetz sieht dennoch keine expliziten Qualifikationen vor (vgl. Kalss und Schimanka 2010, S. 79).

10 Christian Konrad war von 1994 bis 2012 Generalanwalt des Österreichischen Raiffeisenverbandes. Erwin Hameseder war zum Zeitpunkt der Erhebung Generaldirektor der Raiffeisenlandesbank NÖ-Wien und Walter Rothensteiner Vorstandsvorsitzender der RZB. 
Abb. 1: Gesamtzahl und Anzahl der parteinahen Multiaufsichtsräte zwischen 1989 und 2011

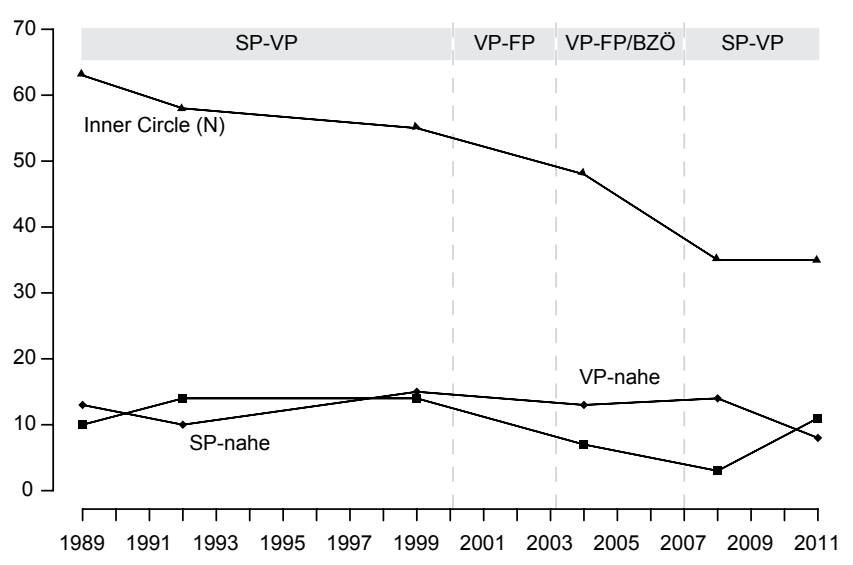

,zum einen das enorme Privatisierungstempo und - damit zusammenhängend - der Primat des Schuldenabbaus binnen einer Legislaturperiode, zum anderen die Vollprivatisierung der im Portfolio der ÖIAG versammelten Unternehmen“ (Obinger 2006, S. 164). 2000 wurden die Restanteile (41,1\%) an der Austria Tabak sowie $100 \%$ am Dorotheum veräußert. Zwischen 2000 und 2005 folgten Vollprivatisierungen eines der derzeit führenden Edelstahl-Herstellers Böhler-Uddeholm sowie der Voest-Alpine-Töchter VA Tech und VA Stahl.

Eines der Hauptergebnisse ist, dass sich die ,Wendezeit“ in Veränderungen der Zusammensetzung parteinaher Multiaufsichtsräte $(N=80)$ widerspiegelt (s. Abb. 1). Diese machen über den ganzen Zeitraum rund 40,2\% aller Multiaufsichtsräte $(N=199)$ aus.

Dass ein Regierungswechsel „Umfärbungen“ des inner circle zur Folge haben kann, verdeutlicht der Niedergang des SPÖ-Netzwerkes während der Wendezeit (vgl. Abb. 1). Der Rechtswissenschaftler Doralt (2010, S. 52) kommentiert in diesem Zusammenhang die Bestellung von Aufsichtsräten bei Beteiligung der öffentlichen Hand folgendermaßen:

Bis zum Jahr 2000 kann man von einem gemilderten Parteienproporz sprechen. Abweichend davon kam es im Jahr 2000 ausschließlich zur Bestellung von Personen, die der FPÖ und der ÖVP (also den Parteien der kleinen Koalition) nahe standen. Zunächst schieden kraft Gesetzes alle bisherigen Mitglieder mit Ablauf der Hauptversammlung [...] aus. Damit wurden automatisch alle SPÖ-nahen Aufsichtsratsmitglieder eliminiert, ebenso aber auch ÖVP-nahe Aufsichtsratsmitglieder, die großkoalitionär eingestellt waren und für eine starke Sozialpartnerschaft eintraten.

Die vorliegende Studie bestätigt diese Einschätzung. 2004 finden sich bis auf Helmut Draxler (AR OMV), Karl Samstag (AR BA-CA) und Johann Sereinig (Vors. Verbund) keine ,roten“ Manager im inner circle. Nach der ,Wende-Regierung“ verdrängt wiederum die SPÖ die ÖVP aus den Aufsichtsräten. Das kann als klarer Hinweis auf die Persistenz des Proporzes im Kern der „Österreich AG“ gewertet werden. „Rote“ Manager gehen jedoch nicht mehr aus den traditionellen Kaderschmieden der Sozialdemokratie hervor. In der Vergangenheit waren es etwa die „Arbeiterkämmerer“ Caspar Einem, Ferdinand Lacina oder Oskar Grünwald, die zumeist im leitenden Management der OMV vertreten waren. Auch der Bund sozialdemokratischer Akademiker (BSA) brachte einige 
führende Manager hervor (z. B. Alfred Düsing, Siegfried Sellitsch). Beide Rekrutierungsreservoirs scheinen mittlerweile ausgetrocknet zu sein.

In den ersten zehn Jahren des Beobachtungszeitraums (1989 bis 1999), die annähernd durch Parität gekennzeichnet sind, lassen sich hingegen zahlreiche SPÖ-nahe Manager in den Vorstandsetagen ausmachen. Zu nennen sind: Hermann Egger (Kelag), Walter Fremuth (Verbund), Anton Heschgl (Austrian Airlines), Herbert Schröfelbauer (Ö. Donaukraftwerke), Klaus Stadler (Wiener Holding). Insbesondere für Aufsichtsräte staatsnaher Unternehmen dieser Zeit gilt, was ein deutscher Korrespondent über die AUA schrieb: „Als die Österreichische Volkspartei (ÖVP) und die Sozialisten (SPÖ) vor zwölf Jahren das Unternehmen gründeten, sicherten sie sich nach Koalitionsbrauch je die Hälfte der Posten vom Aufsichtsrat bis zur Putzfrau“ (Der Spiegel 1969).

Die gleichmäßige Vertretung der Großparteien im inner circle ist jedoch nur zum Teil auf das Proporzabkommen in staatsnahen Unternehmen zurückzuführen. Ein weiterer gewichtiger Grund ist die politische Aufladung des Bankenwesen, von der beide Großparteien gleichermaßen profitierten (vgl. Dirninger 2010). SPÖ-nahe (Androsch, Randa, Sellitsch) ${ }^{11}$ und ÖVP-nahe Banker (Drenning, Petrak, Schmidt-Chiari) ${ }^{12}$ sowie die im Aufsichtsrat der großen Finanzunternehmen vertretenen Spitzenmanager beider Couleur (SPÖ: Kirchweger, Hollweger, ÖVP: Rauch, Igler) ${ }^{13}$ hielten sich die Waage. Es kann von tendenziell ,roten“" und „schwarzen“ Bankengruppen gesprochen werden.

In der ,roten“ Bank Austria war bis 2000 die AVZ (Anteilsverwaltung Zentralsparkasse) der größte Aktionär. Alle wichtigen Entscheidungen innerhalb der AVZ wurden vom Sparkassenrat getroffen, dem acht Mitglieder angehörten, darunter fünf aus dem Wiener Gemeinderat und drei aus dem Betriebsrat der Bank. Da in Wien stets die Sozialdemokratie regierte, war der Einfluss der SPÖ auf die Bank rechtlich abgesichert. Das Profil (1994) ordnet Banker wie Karl Vak, Alfons Haiden, Herbert Lugmayr und Gerhard Randa dem Typus „Zler“ zu: „der aggressive, hemdsärmelige Macher, dem nichts ferner liegt als noble Zurückhaltung, als dünkelhaftes Banker-Standesbewußtsein. Der Zler als personifizierter Wiener Gegensatz zum großbürgerlichen Bankier“.

1990 fusionierte die Zentralsparkasse der Gemeinde Wien (,Z“) mit der Länderbank zur Bank Austria und erweiterte damit auch ihre Anzahl an Industriebeteiligungen (Waagner-Biro, Perlmooser Zementwerke).

Auf der anderen Seite stand die „schwarze Reichshälfte“ im Finanzwesen, der die CA sowie die Erste Bank der österreichischen Sparkassen zuzurechnen waren. In diesen

11 Hannes Androsch war Finanzminister (1970-1981) und Generaldirektor der Creditanstalt (1981-1988). Siegfried Sellitsch stand zwischen 1989 und 2001 an der Spitze der Wiener Städtischen Allgemeinen Versicherung.

12 Manfred Drenning gehörte dem Vorstand der Länderbank an und Walter Petrak u. a. jenem der Bundesländer Versicherung.

13 Richard Kirchweger war ein Industriemanager mit Spitzenpositionen in der ÖIAG, der staatlichen Voest und Chemie Linz. Karl Hollweger fungierte als Mitglied der Perlmooser Zementwerke und Vorsitzender des Vorstand der ÖIAG (1994-1999). Franz Rauch war lange Jahre Geschäftsführer des gleichnamigen Fruchtsaftherstellers. Hans Igler hielt mehrere Positionen in der Verstaatlichten Industrie und war Präsident der Österreichischen Industriellenvereinigung (1972-1980). 
Finanzinstitutionen fanden sich stets überwiegend ÖVP-nahe Manager. ${ }^{14}$ Die CA war historisch gesehen Bank und Industrieholding zugleich (vgl. Stiefel 1983). Zu den Beteiligungen zählten unter anderem: Donau Chemie, Semperit (Chemieindustrie), Universale Baugesellschaft (Bauindustrie), Steyr-Daimler-Puch (Fahrzeugindustrie) und Wienerberger (Ziegelhersteller). Die zentrale Stellung der Großbanken in der Volkswirtschaft räumte den Bankern eine Monopolstellung in den AR-Vernetzungen ein, virtuosenhaft nutzten sie das Netzwerk zur Pflege von Außenbeziehungen.

In den späten neunziger Jahren setzte jedoch eine Entpolitisierung des Bankenwesens ein. 1997, am Ende eines nur schleppend vorankommenden und durch politische Interventionen gekennzeichneten Privatisierungsprozesses der CA (vgl. Grubelnik 1998, S. 193 ff.), kam es zu der bislang am weitesten reichenden Umwälzung im österreichischen Bankenwesen. Die Bank Austria kaufte die CA, ${ }^{15}$ und nach der gesellschaftsrechtlichen Verschmelzung wurde die Unternehmensstrategie der CA, den vererbten Bauchladen an Firmenbeteiligungen abzubauen, zügig fortgesetzt. Der neue Bankriese koppelte sich zunehmend von der Politik ab und wurde zuerst durch die Bayerischen Hypo- und Vereinsbank und später durch die italienischen Unicredit übernommen.

Die konservative Reichshälfte verlor vor allem durch das Ende der Creditanstalt an Zugriff in der Wirtschaft. Zudem erfuhr der überwiegend traditionell von ÖVP-nahen Spitzenmanagern geleitete Sparkassensektor mit dem Spitzeninstitut Erste Bank eine politische Entladung. Aktien der „Ersten Group“ werden heute nicht nur in Wien, sondern auch in Prag und Bukarest gehandelt - lokale Verwurzelungen verlieren folglich an Bedeutung.

All diese Umbrüche zeigen, dass neben Privatisierungen von Industriebetrieben die Unternehmenskonzentrationen sowie Internationalisierungen der Finanzinstitute (Dirninger 2010; Hofstätter und Korom 2012) und das Ende der Bank-Industrie-Beziehungen eine schrittweise Erosion des gesamtösterreichischen Unternehmensnetzwerkes auslösten. In einem kumulativen Effekt führten diese Trends auch dazu, dass der inner circle auf nur 35 Netzwerkvirtuosen im Jahr 2008 schrumpfte (vgl. Tab. 1).

\subsection{Politisierte Manager als Aufsichtsrat-,Kumulierer“}

Mandatkumulation in der Wirtschaft kann diverse Formen annehmen. Hier sollen zwei Aufsichtsratstypen im inner circle unterschieden werden: der Mehrfachaufsichtsrat mit und jener ohne Vorstandsmandat. Aus dem zeitlichen Verlauf in Abb. 2 wird deutlich, dass ab 1999 mehr ÖVP-nahe als SPÖ-nahe Vorstände zu finden sind. Das ist im Wesentlichen auf den Aufstieg der Raiffeisen-Bankengruppe und ihrer Führungskräfte konservativer Couleur zurückzuführen. Die Raiffeisen-Zentralbank (RZB) ist zur Zeit die größte Beteiligungsgesellschaft Österreichs. Die Holding hält milliardenschwere Beteiligungen

14 Von 1936 bis 1959 stand unter drei politischen Systemen („Ständestaat“, NS-Herrschaft, Zweite Republik) der fest im Wirtschaftsbund der ÖVP verankerte Manager Josef Joham der CA vor.

15 Diese Übernahme wurde überwiegend als parteipolitisch motiviert angesehen und störte den Hausfrieden in der großen Koalition empfindlich. Nach Einschätzung des damaligen Finanzministers Lacina ist sogar die Aufkündigung der Zusammenarbeit zwischen SPÖ und ÖVP im Jahre 2000 zumindest teilweise auf den Verkauf der CA an die Bank Austria und den damit einhergehenden politischen Konflikt zurückzuführen (Lacina 2007, S. 466). 


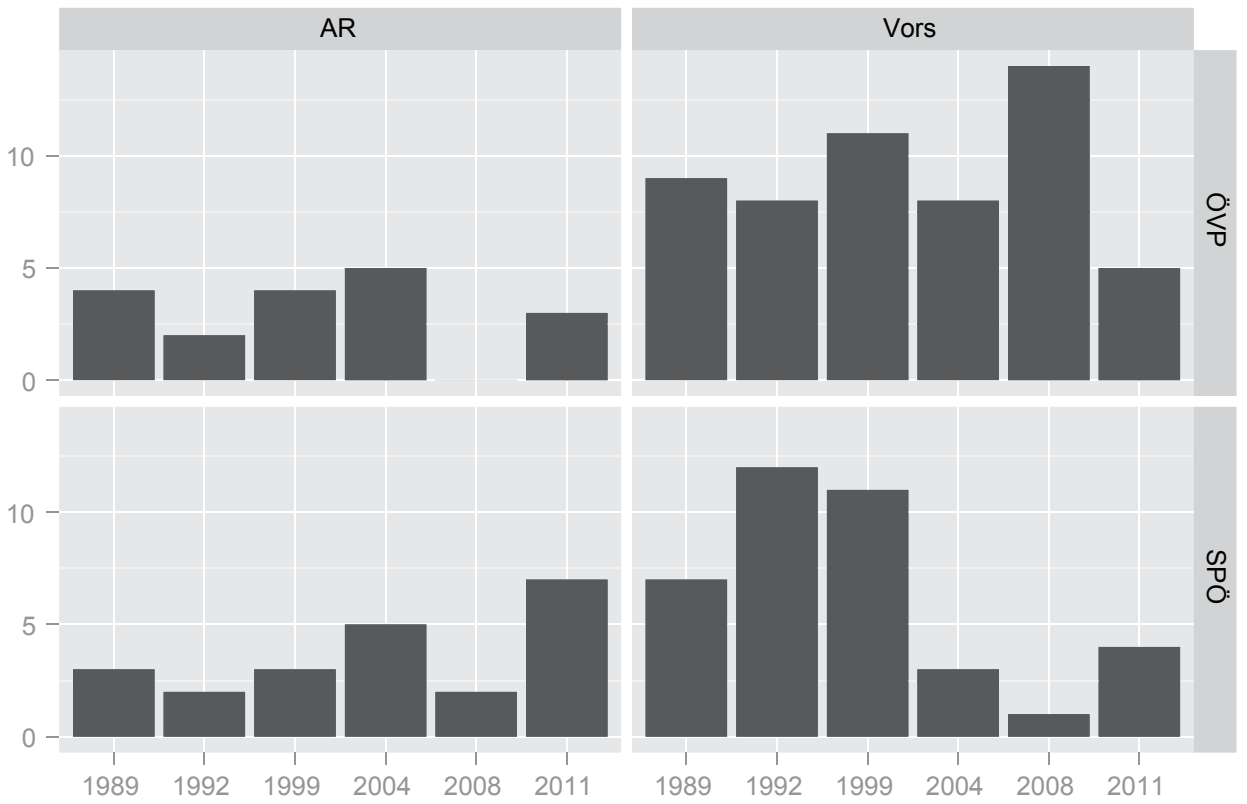

Abb. 2: Zwei Typen von Multiaufsichtsräten im Zeitverlauf (1989-2011)

an dem Baukonzern Strabag, den Nahrungsmittelkonzernen Agrana und Leipnik, dem Medienverlag Kurier sowie dem Versicherungsunternehmen Uniqa. Die jeweiligen Landesbanken gelten als wichtigste Geldgeber in den Bundesländern Oberösterreich und Niederösterreich. Im Zuge der Privatisierung des Flaggschiffs der ehemals verstaatlichten Industrie, dem Stahlkonzern Voest, war es die RLB OÖ, die große Teile der neu entstandenen Privatunternehmen aufkaufte. Es kann daher behauptet werden, dass der ,grüne Riese" als weit verästelte Unternehmensgruppe an die Stelle der ehemals verstaatlichten Banken (CA, Länderbank) getreten ist (Korom 2012). Das Raiffeisen-Topmanagement gehört überwiegend der katholischen, farbentragenden Studentenverbindung CV (Cartellverband) an und ist für seine guten Verbindungen zur ÖVP bekannt. Die politische Haltung dieser Führungskräfte geht aus folgender Interviewpassage hervor (Die Presse 2012):

Interviewer: Wie politisch ist Raiffeisen? Sind Sie ÖVP-Mitglied?

Erwin Hameseder [2012 zum Obmann des Raiffeisenverbandes gewählt]: Ich habe noch nie einen Kunden oder einen Mitarbeiter gefragt, welcher Partei er angehört. Das wäre völlig absurd. Ich persönlich habe eine klare politische Einstellung, zu der ich stehe. Ich bin ÖVP-Mitglied.

Im Gegensatz dazu gehört das rote Bankenmanagement - sieht man von der ÖNB ab der Vergangenheit an. Noch 1999 konnte man unter der Wirtschaftselite führende SPÖBanker finden. Der BAWAG-Generaldirektor Walter Flöttl galt z. B. als „Botschafter des offiziellen Kunstverständnisses der SPÖ““ (vgl. Falter 2008). Heute sind Karl Samstag und Erich Hampel die einzigen verbliebenen „,roten“ Generaldirektoren, die in ihrem Ruhestand noch die eine oder andere Aufsichtsratsfunktion ausüben. 
Dass die Netzwerkvirtuosen der österreichischen Wirtschaft nach wie vor durch ein politisches Profil gekennzeichnet sind, ist zum Gutteil auf „AR-Kumulierer“ ohne Vorstandsmandat in staatsnahen Unternehmen wie der OMV, den Österreichischen Lotterien, den Wiener Stadtwerken, der ÖBB, den Casinos Austria und den regionalen Energieversorgern zurückzuführen.

Es sind in jüngster Zeit nahezu ausschließlich von der Politik gesteuerte Aufsichtsratsbesetzungen in staatsnahen Dienstleistungsunternehmen (z. B. Verbund), die den Querbeziehungen zwischen Politik und Wirtschaft Kontinuität verleihen. In der Vergangenheit hingegen ging eine etatistisch-korporatistische Wirtschaftsordnung mit parteinahen Bankdirektoren (z. B. Hannes Androsch, CA) und statesmen of industry in den wesentlichen Schaltzentralen der Verstaatlichten (z. B. Peter Strahammer, Voest) einher. Diese agierten an der Schnittstelle zwischen Wirtschaft und einer Politik, die sich ihre Eigentümerinteressen in staatsnahen Unternehmen sicherte. ${ }^{16}$ Mit zunehmender Privatisierung gingen die personellen Querbeziehungen zwischen den Spitzen beider Systeme und damit ein potentielles Koordinationsmittel verloren.

\subsection{Machtasymmetrie zwischen Managern und Parteien - das Erbe der Verstaatlichten}

Multiaufsichtsräte machen nur einen Bruchteil aller in den größten 125 Unternehmen Österreichs tätigen Spitzenmanager aus. In Abb. 3 wurden diese Unternehmen nach der Parteizugehörigkeit aller registrierten Multiaufsichtsräte klassifiziert. Waren in einem Unternehmen sowohl ,rote“ als auch ,schwarze“ Manager vertreten, so wurde das Unternehmen als „rot-schwarz“ eingeordnet. Es zeigt sich, dass bis 1999 die Mitglieder des inner circle Managerpositionen in rund 40\% aller Unternehmen einnahmen. In nachfolgenden Jahren geht der Anteil auf 30\% zurück. Abbildung 3 kann auch entnommen werden, dass der Proporz (gemessen an dem Anteil aller rot-schwarzen Unternehmen) während der ,Wendezeit“" eine untergeordnete Rolle spielte. 2011 findet sich eine Farbenkonstellation wieder, die jener der 1990er Jahre ähnelt.

Im Folgenden sollen jene ausgewählten Unternehmen genauer betrachtet werden, in denen Multiaufsichtsräte tätig waren. Falls es sich bei diesen um staatsnahe Großbetriebe handelt, kann von einer Machtasymmetrie zwischen Manager und Partei ausgegangen werden: In ihrer Rolle als Eigentumsvertreter können sich Parteien für oder gegen Kandidaten für den Aufsichtsrat entscheiden. Aufgrund dieser Abhängigkeit der Manager sprechen Experten für Unternehmensrecht auch von „Politikgünstlingen“ in den Kontrollorganen staatsnaher Unternehmen (vgl. Kraßnig 2010, S. 15).

Unter dem Begriff ,staatsnahe Unternehmen“ sollen hier alle jene Firmen erfasst werden, an denen zum Zeitpunkt des Vorstands- oder Aufsichtsratsmandats eines Managers der Bund, die Länder, die ÖIAG, der ÖGB oder die verstaatlichten Banken (Creditanstalt, Länderbank) mehrheitlich beteiligt waren. Als Kriterium dafür, ob ein Manager in einer machtasymmetrischen Beziehung zur Partei gestanden ist, soll eine in offiziellen Lebens-

16 Einer solchen Personalunion waren Konflikte inhärent. Berühmt wurden Aussprüche des VoestGeneraldirektors Heribert Apfalter, der Repräsentanten der ÖIAG als „russische Bürokraten“ mit Praxisferne bezeichnete (Profil 2005). 
Abb. 3: Die politische Einfärbung der 125 größten Unternehmen nach Parteizugehörigkeit der Multiaufsichtsräte (1989-2011)

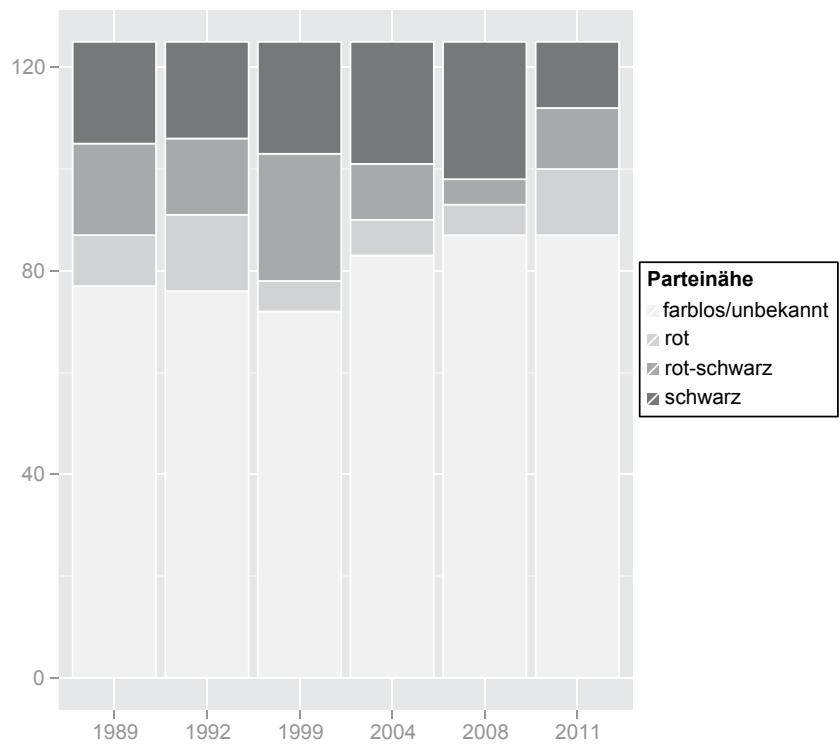

läufen aufscheinende Angabe über einen zumindest fünf Jahre andauernden Berufsabschnitt in einem staatsnahen Unternehmen dienen.

Wendet man ein derartiges Kriterium zur Messung von Abhängigkeiten in Manager-Parteien-Netzwerken an, so fällt der empirische Nachweis von Machtasymmetrien zugunsten der Parteien eindeutig aus: Nur neun der insgesamt 80 parteinahen Multiaufsichtsräte im Sample weisen gänzlich in der Privatwirtschaft verlaufende Karrieren auf. Dabei handelt es sich um die Raiffeisen-Manager Georg Doppelhofer, Erwin Hameseder, Christian Konrad, Peter Püspök, Walter Rothensteiner, Ludwig Scharinger, Christian Teufl sowie um den Banker Andreas Treichl und den Industriellen Manfred Mautner Markhof.

Dieser Befund muss jedoch relativiert werden. Wie gezeigt, waren Spitzenmanager auch in der Hochblüte der Verstaatlichten keine der Politik gänzlich untergeordneten Parteisoldaten (vgl. Kap. 2.1). Des Weiteren erweiterten sich die Freiheitsgrade der Manager im Laufe der Privatisierung (vgl. Kap. 2.2). Das soll anhand eines repräsentativen Beispiels illustriert werden: Die ehemalige Abgeordnete zum Wiener Landtag Maria Schaumayer (1938-2013) wechselte 1982 in den Finanzvorstand des ÖIAG-Unternehmens OMV. Sie initiierte dort eine Teilprivatisierung, die den Einfluss der Politik wesentlich schmälern sollte. Nach einer fünfjährigen Amtsperiode (1990-1995) als Präsidentin der Österreichischen Nationalbank wurde Schaumayer ab 2000 Regierungsbeauftrage für die Entschädigung der Zwangsarbeiter unter dem NS-Regime. Schaumayers Karriere verlief folglich nahezu gänzlich im staatsnahen Bereich, jedoch avancierte sie zur (einzigen) Grande Dame der ÖVP. Sie begegnete Spitzenpolitikern auf Augenhöhe und wurde 2012 sogar von der ÖVP beauftragt, einen Verhaltenskodex für die Partei zu entwerfen. Damit wurde sie als moralische Autorität über alle Politiker gestellt.

Derartige Verschiebungen in der Machtbalance machen deutlich, dass mit Ende des öffentlichen Wirtschaftssektors und damit dem Rückgang von Patronageressourcen eine Enthierarchisierung der Parteien-Manager-Verflechtungen eingesetzt hat. Die große Zahl 
an beobachteten Machtasymmetrien ist daher weitgehend als Erbe der Verstaatlichten zu werten. Inwiefern die neuen, stärker ausbalancierten Manager-Parteien-Beziehungen, wie sie z. B. zwischen Raiffeisen und der ÖVP zu finden sind, interessengesteuert sind, lässt sich empirisch nicht verlässlich ermitteln.

\section{Schlussfolgerungen}

Proporz (Typ 1), interessenbasierter Austausch (Typ 2), politische Manager mit Karriereambitionen (Typ 3) - das sind die drei Kategorien, mit denen Parteien-Manager-Verflechtungen erfasst werden können. Welcher dieser Beziehungstypen kennzeichnet nun den österreichischen Fall?

Nicht nur aus historischer Perspektive kommt dem Proporz eine große Bedeutung zu (Müller 2006). Insbesondere wenn es um Ämterpatronage innerhalb der Eliten geht, erweisen sich alte Personalpolitiken als relativ persistent (Treib 2012). Das verdeutlicht am erhellendsten der Nachweis, dass die Ab- oder Zunahme von Partei-Mandataren in der Regierung entsprechende Umfärbungen im Führungspersonal staatlicher Großbetriebe zur Folge hat (vgl. Ennser-Jedenastik 2013). Auch unsere Studie konnte derartige tektonische Verschiebungen entlang von Parteilinien innerhalb des inner circle während der „Wenderegierung“" nachweisen.

Dabei ist jedoch in vielen Fällen ein Wandel der ursprünglich asymmetrischen Patron-Klient-Beziehungen anzunehmen. So kann etwa der ehemalige Generaldirektor Erich Hampel (BA), der derzeit dem Aufsichtsrat der staatlichen Post angehört, nicht mehr treffend als abhängiger Klient bezeichnet werden. Hampel ist zwar wie zahlreiche andere Manager, deren Karriere in die Zeit der Verstaatlichten zurückreicht, weiterhin in Parteiennetze eingebunden. Er könnte jedoch diese aufkündigen, ohne großen Schaden zu nehmen. An die Stelle der ursprünglichen Machtasymmetrie ist ein reziproker Nutzen getreten: Parteien können erfahrenen Führungskräften in den Kontrollgremien staatlicher Betriebe vertrauen, Manager erhoffen sich von diesen AR-Mandaten Marktvorteile für das Unternehmen oder eine interessante Beschäftigung (am Ende ihrer Karriere). In welchem Ausmaß Typ 2 und 3 die herkömmlichen Partei-Manager-Netzwerke abgelöst haben, ist nicht eruierbar.

Des Weiteren muss berücksichtigt werden, dass Proporz (Typ 1) nicht mehr für denselben Managertypus steht. In den 1990er Jahren hatten noch so genannte statesmen of industry wie etwa Oskar Grünwald zentrale Positionen in der „Österreich AG“. Sie konnten zum Typ „Organisierungselite“ gerechnet werden, ,,deren Leistung im Aufbau kollektiver Verpflichtungs- und Kompromissfähigkeit und in der Vermittlung zwischen konfligierenden Interessen“ stand (Streeck 2006, S. 165 f.). An ihrer Stelle traten stärker am Profit (und nicht etwa am sozialen Ausgleich) interessierte „Organisationseliten“ mit loseren Parteienaffiliationen. Zumeist handelt es sich dabei um Aufsichtsrat-,,Kumulierer“, deren Einfluss auf Unternehmensstrategien gering sein dürfte.

Trotz dieser Entwicklungen ist das politische Kapital über zwei Jahrzehnte bis heute ein Merkmal der zentralen Aufsichtsräte in Österreichs Wirtschaft geblieben. Selbst der Untergang großer politisierter Wirtschaftsbastionen im Bankenwesen (Creditanstalt Bank Austria, BAWAG) führte nicht zu einer gänzlichen Entpolitisierung von Österreichs Topmanagement. Ziel dieses Aufsatzes war es nicht nur, diese relative Konstanz her- 


\begin{tabular}{|c|c|}
\hline SPÖ-nahe Multiaufsichtsräte & ÖVP-nahe Multiaufsichtsräte \\
\hline Hannes Androsch & Ulrike Baumgartner-Gabitzer \\
\hline Otto Binder & Erich Becker \\
\hline Helmut Draxler & Markus Beyrer \\
\hline Hermann Egger & Georg Doppelhofer \\
\hline Helmut Elsner & Manfred Drenning \\
\hline Walter Flöttl & Gerhard Falch \\
\hline Walter Fremuth & Rudolf Gruber \\
\hline Alois Froschauer & Josef Halbmayr \\
\hline Hedwig Fuhrmann & Erwin Hameseder \\
\hline Oskar Grünwald & Burkhard Hofer \\
\hline Rene Alfons Haiden & Hans Igler \\
\hline Erich Hampel & Hellmuth Klauhs \\
\hline Anton Heschgl & Christian Konrad \\
\hline Karl Hollweger & Stephan Koren \\
\hline Heinrich Horny & Klaus Liebscher \\
\hline Herbert Kasser & Andreas Maurer \\
\hline Christian Kern & Manfred Mautner-Markhof \\
\hline Richard Kirchweger & Siegfried Meysel \\
\hline Max Kothbauer & Peter Mitterbauer \\
\hline Franz Lauer & Ewald Nageler \\
\hline Herbert Lugmayr & Walter Nettig \\
\hline Werner Muhm & Walter Petrak \\
\hline Gabriele Payr & Franz Pinkl \\
\hline Horst Pöchhacker & Peter Püspök \\
\hline Gerhard Randa & Josef Ratzenböck \\
\hline Karl Samstag & Franz Rauch \\
\hline Herbert Schröfelbauer & Walter Rothensteiner \\
\hline Richard Seiser & Ludwig Scharinger \\
\hline Siegfried Sellitsch & Erhard Schaschl \\
\hline Johannes Sereinig & Maria Schaumayer \\
\hline Klaus Stadler & Richard Schenz \\
\hline Josef Staribacher & Herbert Schimetschek \\
\hline Peter Strahammer & Guido N. Schmidt-Chiari \\
\hline Rudolf Streicher & Wilfried Stadler \\
\hline Herbert Tumpel & Karl Stoss \\
\hline Günter Weninger & Josef Taus \\
\hline Helmut Zilk & Christian Teufl \\
\hline \multirow[t]{5}{*}{ Peter Zöllner } & Andreas Treichl \\
\hline & Leo Wallner \\
\hline & Erwin Wenzl \\
\hline & Leopold Windtner \\
\hline & Hannes Zach \\
\hline
\end{tabular}

Quelle: Eigene Datenbank 
auszuarbeiten, sondern auch die gewandelten Bedeutungen von Parteien-Manager-Verflechtungen darzustellen.

\section{Literatur}

Aiginger, Karl. 1991. Industriepolitik. In: Handbuch des politischen Systems Österreichs, Hrsg. Herbert Dachs, 513-524. Wien: Manz.

Aiginger, Karl. 1999. The privatization experiment in Austria. Austrian Economic Quarterly 4 (4): 261-270.

Belke, Ansgar, und Friedrich Schneider. 2006. Privatization in Austria: Some theoretical reasons and performance measures. In: Privatization experiences in the European Union, Hrsg. Marko Köthenbürger, Hans-Werner Sinn, und John Whalley, 89-116. Cambridge: MIT Press.

Böheim, Michael, Heinz Handler, und Margit Schratzenstaller. 2010. Optionen einer einnahmensbasierten Budgetkonsolidierung. WIFO-Monatsberichte 3:269-283.

Bond, Matthew, Siana Glouharova, und Nicholas Harrigan. 2010. The political mobilization of corporate directors: Socio-economic correlates of affiliation to European pressure groups. British Journal of Sociology 61 (2): 306-335.

Bourdieu, Pierre. 1998. Praktische Vernunft. Zur Theorie des Handelns. Frankfurt a. M.: Suhrkamp.

Burris, Val, und James Salt. 1990. The politics of capitalist class segments: A test of corporate liberalism theory. Social Problems 37 (3): 341-359.

Dirninger, Christian. 2010. Transformationen im österreichischen Bankensystem seit Mitte der 1950er Jahre. In: Strukturwandel und Internationalisierung im Bankwesen seit den 1950er Jahren, Hrsg. Ralf Ahrens und Harald Wixforth, 141-173. Stuttgart: Steiner.

Dobler, Helmut. 1983. Der persistente Proporz: Parteien und verstaatlichte Industrie. In: Zwischen Koalition und Konkurrenz. Österreichs Parteien seit 1945, Hrsg. Peter Gerlich und Wolfgang C. Müller, 319-333. Wien: Braumüller.

Doralt, Peter. 2010. Erscheinungsformen des Aufsichtsrats und ihr Zusammenhang mit den Ursachen seines Versagens. Ein erster Versuch. In: Handbuch für den Aufsichtsrat, Hrsg. Susanne Kalss und Peter Kunz, 41-63. Wien: Facultas.

Ennser-Jedenastik, Laurenz. 2013. Die parteipolitische Besetzung von Spitzenfunktionen in österreichischen Staatsunternehmen: eine quantitative Analyse. Österreichische Zeitschrift für Politikwissenschaft 42 (2): 125-143.

Fehr, Ernst, und Alexander Van der Bellen. 1982. Aufsichtsräte in öffentlichen Unternehmen. Skizzen zur politischen Ökonomie Österreichs. Zeitschrift für öffentliche und gemeinwirtschaftliche Unternehmen 5 (2): 123-150.

Grant, Wyn. 1987. Business and politics in Britain. Basingstoke: Macmillan.

Grubelnik, Klaus. 1998. Die rote Krake. Eine Bank erobert Österreich. Wien: Molden.

Hofstätter, Lukas, und Philipp Korom. 2012. Vom Austrokorporatismus zum Austrokapitalismus: Das Beispiel der Entbettung österreichischer Banken. In: Entfesselte Finanzmärkte? Soziologische Analysen des modernen Kapitalismus, Hrsg. Klaus Kraemer und Sebastian Nessel, 161-180. Frankfurt a. M.: Campus.

Höpner, Martin. 2006. Beiträge der Unternehmen zur Parteienfinanzierung: Wer spendet an wen? Und warum? Zeitschrift für Parlamentsfragen 37 (2): 293-311.

Kafka, Gustav E. 1962. Österreichs gelähmte Regierung. Wort und Wahrheit 17: 590-612.

Kalss, Susanne, und Matthias Schimka. 2010. Qualifikationsanforderungen an die AR-Mitglieder. In: Handbuch für den Aufsichtsrat, Hrsg. Susanne Kalss und Peter Kunz, 65-90. Wien: Facultas.

Koren, Stephan. 1964. Sozialisierungsideologie und Verstaatlichungsrealität. In: Die Verstaatlichung in Österreich, Hrsg. Wilhelm Weber, 9-340. Berlin: Duncker \& Humblot. 
Korom, Philipp. 2012. Kein Ende der „Österreich AG“? Über die Beständigkeit eines koordinierten Unternehmensnetzwerkes in Zeiten von Privatisierung und Internationalisierung. Österreichische Zeitschrift für Politikwissenschaft 41 (2): 141-160.

Kraßnig, Ulrich. 2010. Der Aufsichtsrat staatsnaher Unternehmen im Zangengriff der Politik. Aufsichtsrat aktuell 4: 12-15.

Lacina, Ferdinand. 2007. Umstrukturierung im Bankensektor - Politik und Geschäft. In: Österreichs Kreditwirtschaft. Von der Reichsmark über den Schilling zum Euro, Hrsg. Erwin Frasl, René A. Haiden, und Josef Taus 463-469. Graz: NWV.

Mathis, Franz. 1995. Zwischen Lenkung und freiem Markt. In: Österreich in den Fünfzigern, Hrsg. Thomas Albrich, et al., 169-180. Innsbruck: Österreichischer Studien.

McMenamin, Iain, und Roger Schoenmann. 2007. Together forever? Explaining exclusivity in party-firm relations. Political Studies 55 (1): 153-173.

Meth-Cohn, Delia, und Wolfgang C. Müller. 1994. Looking reality into the eye: The politics of privatization in Austria. In: Industrial privatization in western Europe. Pressures, problems, and paradoxes, Hrsg. Vincent Wright, 160-179. London: Pinter.

Mizruchi, Mark S. 1992. The structure of corporate political action: interfirm relations and their consequences. Cambridge: Harvard University Press.

Müller, Wolfgang C. 1988. Patronage im österreichischen Parteiensystem. Theoretische Überlegungen und empirische Befunde. In: Das österreichische Parteiensystem, Hrsg. Anton Pelinka und Fritz Plasser, 457-487. Wien: Böhlau.

Müller, Wolfgang C. 2006. Party patronage and party colonialization of the state. In: Handbook of Party Politics, Hrsg. Richard S. Katz und William Crotty, 189-195. London: Sage.

Nee, Victor, und Sonja Opper. 2007. On politicized capitalism. In: On capitalism, Hrsg. Victor Nee und Richard Swedberg, 93-127. Stanford: Stanford University Press.

Obinger, Herbert. 2006. „Wir sind Voesterreicher“: Bilanz der ÖVP/SPÖ-Privatisierungspolitik. In: Schwarz-blau. Eine Bilanz des „Neu-Regierens“, Hrsg. Emmerich Tálos, 154-169. Wien: Lit.

Pelinka, Anton, und Sieglinde Rosenberger. 2000. Österreichische Politik. Grundlagen, Strukturen, Trends. Wien: WUV-Universitätsverlag.

Sickinger, Hubert. 2011. Politische Korruption und der Wandel der Rahmenbedingungen für politische Skandale in der Zweiten Republik. In: Korruption in Österreich. Historische Streiflichter, Hrsg. Ernst Bruckmüller, 111-130. Wien: Braumüller.

Stark, David, und Balazs Vedres. 2012. Political holes in the economy: The business network of partisan firms in Hungary. American Sociological Review 77 (5): 700-722.

Stiefel, Dieter. 1983. The reconstruction of the credit-anstalt. In: International Business and Central Europe, 1918-1939, Hrsg. Alice Teichova, 415-430. Leicester: Leicester University Press.

Stiefel, Dieter. 2011. Verstaatlichung und Privatisierung in Österreich. Illusion und Wirklichkeit. Wien: Böhlau.

Streeck, Wolfgang. 2006. Nach dem Korporatismus: Neue Eliten, neue Konflikte. In: Deutschlands Eliten im Wandel, Hrsg. Herfried Münkler, Grit Straßenberger, und Matthias Bohlender, 149-176. Frankfurt a. M.: Campus.

Streeck, Wolfgang, und Kathleen Thelen. 2005. Introduction: Institutional change in advanced political economies. In: Beyond continuity. Institutional change in advanced political economies, Hrsg. Wolfgang Streeck und Kathleen A. Thelen, 1-39. Oxford: Oxford University Press.

Treib, Oliver. 2012. Party patronage in Austria: From reward to control. In: Party patronage and party government in European democracies, Hrsg. Petr Kopecky, Peter Mair, und Maria Spirova, 31-53. Oxford: Oxford University Press.

Turnheim, Georg. 2009. Österreichs Verstaatlichte. Die Rolle des Staates bei der Entwicklung der österreichischen Industrie von 1918 bis 2008. Wien: Manz.

Useem, Michael. 1984. The inner circle: Large corporations and the rise of business political activity in the U.S. and U. K. New York: Oxford University Press. 
Weber, Fritz. 2011. Verstaatlichung und Privatisierung in Österreich 1946-1986. Zeitschrift für öffentliche und gemeinwirtschaftliche Unternehmen 34 (2): 126-147.

White, Harrison C. 1992. Identity and control. A structural theory of social action. Princeton: Princeton University Press.

Ziegler, Rolf. 1987. Besitzverhältnisse, Parteipräferenz und Personenverbindungen in der österreichischen Wirtschaft. Österreichische Zeitschrift für Soziologie 12 (2): 81-92.

\section{Zeitungsartikel}

Falter. (2008, 9. Juli). Die Genossen Flöttl. http://www.florianklenk.com/2008/07/08/die-genossenflottl/. Zugegriffen: 31. Jan. 2014.

Der Spiegel. (1969, 12. Mai). Bedingt einsatzfähig. http://www.spiegel.de/spiegel/print/d-45741438. html. Zugegriffen: 31. Jan. 2014.

Der Standard. (2011a, 18. Feb.). „Ich bin ein Produkt des Proporzes“ Interview mit Claus J. Raidl. http://derstandard.at/1297818382633/Ich-bin-ein-Produkt-des-Proporzes. Zugegriffen: 31. Jan. 2014.

Der Standard. (2011b, 14. Mai.). „Unsere Politiker sind zu blöd und zu feig“. http://derstandard. at/1304551930329/Treichl-Unsere-Politiker-sind-zu-bloed-und-zu-feig. Zugegriffen: 31. Jan. 2014.

Die Presse. (2012, 21. Juni). „Für ganz links und rechts haben wir null Verständnis“ Interview mit Erwin Hameseder. http://diepresse.com/home/wirtschaft/economist/767925/Hameseder_Furganz-links-und-rechts-null-Verstaendnis. Zugegriffen: 31. Jan. 2014..

Profil. (1994, 11. April). Der Bank-Austria Komplex.

Profil. (2005, 27. Aug.). Vollprivatisierung: Die Voest-Saga. http://www.profil.at/articles/0534/ 560/120145/vollprivatisierung-die-voest-saga. Zugegriffen: 31. Jan. 2014.

The Economist. (2003, 4. Sept.). Don't sell our family silver!

Philipp Korom, Dr. rer. soc. oec. Mag rer. nat. Mag. phil., geb. 1983, wissenschaftlicher Mitarbeiter am Max Planck Institut in Köln; der Artikel wurde 2013 am Institut für Soziologie/Graz abgefasst. Arbeitsschwerpunkte: Wirtschaftssoziologie, Eliten-/Intellektuellensoziologie, soziale Ungleichheit; ausgewählte Publikationen: Die Wirtschaftseliten Österreichs, 2013, Konstanz/München: UVK; Wer wurde als homosexuell verfolgt?, in: Kölner Zeitschrift für Soziologie und Sozialpsychologie, 2012, 64(4), gem. mit Christian Fleck; Kapitalismus und Gender, in: Berliner Journal für Soziologie 2012, 22(1), gem. mit Klaus Kraemer und Sebastian Nessel; Kein Ende der „Österreich AG“?, in: Österreichische Zeitschrift für Politikwissenschaft 2012, 41(2); Der Aufstieg des Expertenintellektuellen, in: SWS-Rundschau 2012, 52(1). 